\title{
Research on the development and application of physical materials
}

\author{
Sujun Cheng ${ }^{1, a, *}$ \\ ${ }^{1}$ College of Physics and Electronic Engineering, Xinxiang University, \\ Xinxiang, 453000. P. R. China \\ aemail:sujun_cheng@126.com
}

Keywords: Material physics, Partial differential equation, Boundary value problem

\begin{abstract}
Material physics is a cross subject of physics and materials science and which is through a variety of physical techniques and physical effects, achieving the synthesis of materials, preparation, processing, modification and application. Material physics and chemistry play a leading role in high technical fields and which is the main factor influencing national economy and technology. In this paper, firstly, the main research content of material physics has been expounded, then discussing the development prospects of the subject. Lastly, we studied a series of boundary value problems in Functionally Graded Materials plain fracture.
\end{abstract}

\section{Introduction}

Material physics is a cross subject of physics and materials science and which is through a variety of physical techniques and physical effects, achieving the synthesis of materials, preparation, processing, modification and application[1]. Research scope includes the synthesis, structure, properties and application of materials, the design of new materials and computer simulation of materials. With the development of science and technology, the material is moving toward miniaturization, functional, intelligent direction. It means that the materials industry will have a big change. The corresponding material products will be along with the change.

Nanometer materials, environmental materials, electronic materials and informational materials are now in popular. Most physical properties of the materials are applied in various fields. Industrial developed countries have to increase the material research and development to a strategic height and regard the material as the priority development areas[2]. President Eisenhower in personally formulate the "national material planning" in the 1950s. Through the plan, the USA is increasing in national strength. Former US President Bill Clinton put forward the implementation of "Technical - economic growth" in 1994. Developing the new material was regarded as one of the important matters. It aims to make the USA remain prosperous in the fields of national economy and science technology. The Japanese government regarded the new materials as the key technology for future. The government put forward a large plan such as Western Europe eureka plan which to make an important contribution for the development of new materials[3]. Some other developed countries also have adjusted their economic and technological policies, especially for the research and development of new materials. It takes the new material as a cutting-edge technology.

\section{Development prospects of material physical}

Aviation material is playing a leading role in the development of modern material science and technology. Inorganic body material and engine material are the most important structural application. It is closely related to material physics and chemistry. For aviation and security considerations, aircraft structure materials possess the characteristic of the lower density, higher strength and high reliability. Aircraft as a whole, it uses a small amount of unstructured materials, such as reducing vibration and noise, sealing, etc. It has put forward higher demands for the development of material chemistry. Aviation industry needs varieties of chemical industry material. Largely using the chemical industry material can reduce the weight of the aircraft. The range is increasing and the speed is improving. 
The body materials include aluminum alloys, titanium alloy, resin matrix composites, etc. It focus on low costs and high performance for resin matrix composites technology and make new composite material has higher performance to price ratio. The European company Airbus put forward for more preparation by using the method of material chemistry and application of carbon fiber composite materials to lose weight to $30 \%$ and reduce the flight costs by $40 \%$ [4]. But carbon fiber composite materials technology can reduce weight as well as the manufacturing cost is higher than metal welding structure cost. Now making the use of the technique stores borrowed from Airbus design can loss weight by $15 \%$ and reduce the cost by $15 \%$. But using the new type of metal welded structure fuselage can loss weight by $10 \%$ and reduce the cost by $20 \%$ [5]. Development the low cost and high performance composite materials is great potential.

\section{Application of material physics}

The functional graded material (FGM) is a new kind of heterogeneous materials and composite materials. In recent years, FGM received widespread attention at home and abroad. At present, FGM has become the front research subject of materials science. FGM is a kind of inhomogeneous body. It is characterized at different areas of the material, the mechanical properties of the material show a gradient continuous change and it can be controlled. In this section, we studied the problems of infinite FGM plate with crack and static fracture mechanics concluded I type, II type, I + II type[6]. Through a series of derivation, we boil down the boundary value problem of partial differential equation.

Assuming isotropic materials, the elastic modulus is $\mathrm{E}, \mathrm{V}$ is a variable which is according to the variable y continuous change for functionally graded materials, we can obtain that,

$$
\left(\begin{array}{c}
\varepsilon_{x} \\
\varepsilon_{y} \\
r_{x y}
\end{array}\right)=\left(\begin{array}{ccc}
\frac{1}{E(y)} & -\frac{v(y)}{E(y)} & 0 \\
-\frac{v(y)}{E(y)} & \frac{1}{E(y)} & 0 \\
0 & 0 & \frac{2[1+v(y)}{E(y)}
\end{array}\right)\left(\begin{array}{c}
\sigma_{x} \\
\sigma_{y} \\
\tau_{x y}
\end{array}\right)
$$

The Function (1) also known as,

$$
\varepsilon_{x}=\frac{1}{E(y)} \sigma_{x}-\frac{v(y)}{E(y)} \sigma_{y}, \varepsilon_{y}=-\frac{v(y)}{E(y)} \sigma_{x}+\frac{1}{E(y)} \sigma_{y}, r_{x y}=\frac{2[1+v(y)]}{E(y)} \tau_{x y}
$$

Then function of stress was recommended as following,

$$
U: \sigma_{x}=\frac{\partial^{2} U}{\partial y^{2}}, \sigma_{y}=\frac{\partial^{2} U}{\partial x^{2}}, \tau_{x y}=\frac{\partial^{2} U}{\partial x \partial y}
$$

We let (3) into (2), we hold that

$$
\varepsilon_{x}=\frac{1}{E(y)} \frac{\partial^{2} U}{\partial y^{2}}-\frac{v(y)}{E(y)} \frac{\partial^{2} U}{\partial x^{2}}, \varepsilon_{y}=\frac{v(y)}{E(y)} \frac{\partial^{2} U}{\partial y^{2}}-\frac{1}{E(y)} \frac{\partial^{2} U}{\partial x^{2}}, r_{x y}=-\frac{2[1+v(y)]}{E(y)} \frac{\partial^{2} U}{\partial x \partial y}
$$

Compatibility equation as follows, 


$$
\frac{\partial^{2} \varepsilon_{x}}{\partial y^{2}}+\frac{\partial^{2} \varepsilon_{y}}{\partial x^{2}}=\frac{\partial^{2} r_{x y}}{\partial x \partial y}
$$

Integrating both sides of the equation (4), we can obtain that

$$
\begin{aligned}
& \frac{\partial^{2} \varepsilon_{x}}{\partial y^{2}}= \frac{1}{E(y)} \frac{\partial^{4} U}{\partial y^{4}}-\frac{v(y)}{E(y)} \frac{\partial^{4} U}{\partial x^{2} \partial y^{2}}-2 \frac{E^{\prime}(y)}{E^{2}(y)} \frac{\partial^{3} U}{\partial y^{3}}-2 \frac{E(y) v^{\prime}(y)-E^{\prime}(y) v(y)}{E^{2}(y)} \frac{\partial^{3} U}{\partial x^{2} \partial y} \\
&+\frac{2 E^{\prime}(y)-E(y) E^{\prime \prime}(y)}{E^{3}(y)} \frac{\partial^{3} U}{\partial y^{2}} \\
&+\frac{2 E^{\prime}(y)\left[E(y) v^{\prime}(y)-E^{\prime}(y) v(y)\right]-E(y)\left[E(y) v^{\prime \prime}(y)-E^{\prime \prime}(y) v(y)\right]}{E^{2}(y)} \frac{\partial^{2} U}{\partial x^{2}} \\
& \frac{\partial_{x y}^{2} r_{x y}}{\partial x \partial y}=-\frac{2[1+v(y)]}{E(y)} \frac{\partial^{4} U}{\partial x^{2} \partial y^{2}}-\frac{2\left(E(y) v^{\prime}(y)-E^{\prime}(y)[1+v(y)]\right)}{E^{2}(y)} \frac{\partial^{3} U}{\partial y^{2} \partial y}
\end{aligned}
$$

We let (6), (7), (8) into (5), we hold that

$$
\begin{aligned}
& \frac{\partial^{4} U}{\partial x^{4}}+2 \frac{\partial^{4} U}{\partial x^{2} \partial y^{2}}+\frac{\partial^{4} U}{\partial y^{4}}-\frac{2 E^{\prime}(y)}{E(y)} \frac{\partial^{3} U}{\partial x^{2} \partial y}-\frac{2 E^{\prime}(y)}{E(y)} \frac{\partial^{3} U}{\partial y^{3}}+ \\
& \frac{2 E^{\prime}(y)\left[E(y) v^{\prime}(y)-E^{\prime}(y) v(y)\right]-E(y)\left[E(y) v^{\prime \prime}(y)-E^{\prime \prime}(y) v(y)\right]}{E^{2}(y)} \frac{\partial^{2} U}{\partial x^{2}} \\
& +\frac{2\left(E^{\prime}(y)\right)^{2}-E(y) E^{\prime \prime}(y)}{E^{2}(y)} \frac{\partial^{2} U}{\partial y^{2}}=0
\end{aligned}
$$

The equation (9) is the basic function of the isotropic functionally graded materials. Then, we study the corresponding boundary conditions of three types.

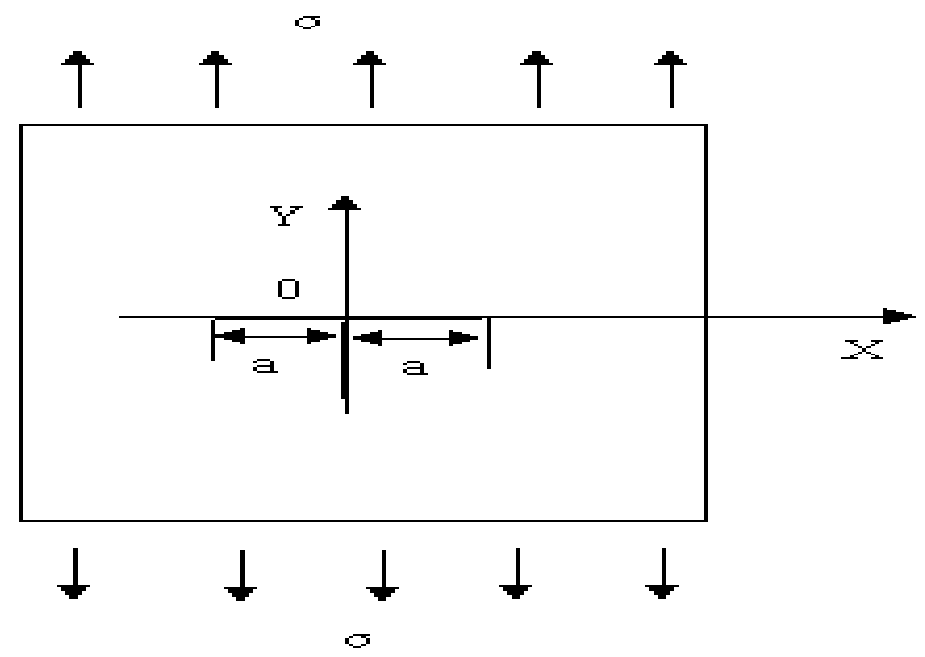

Fig.1 Plate with I mode crack 
As shown in figure 1, the functionally graded material Plate with I mode crack which subjected to symmetric load $\sigma$, its boundary conditions are as follows:

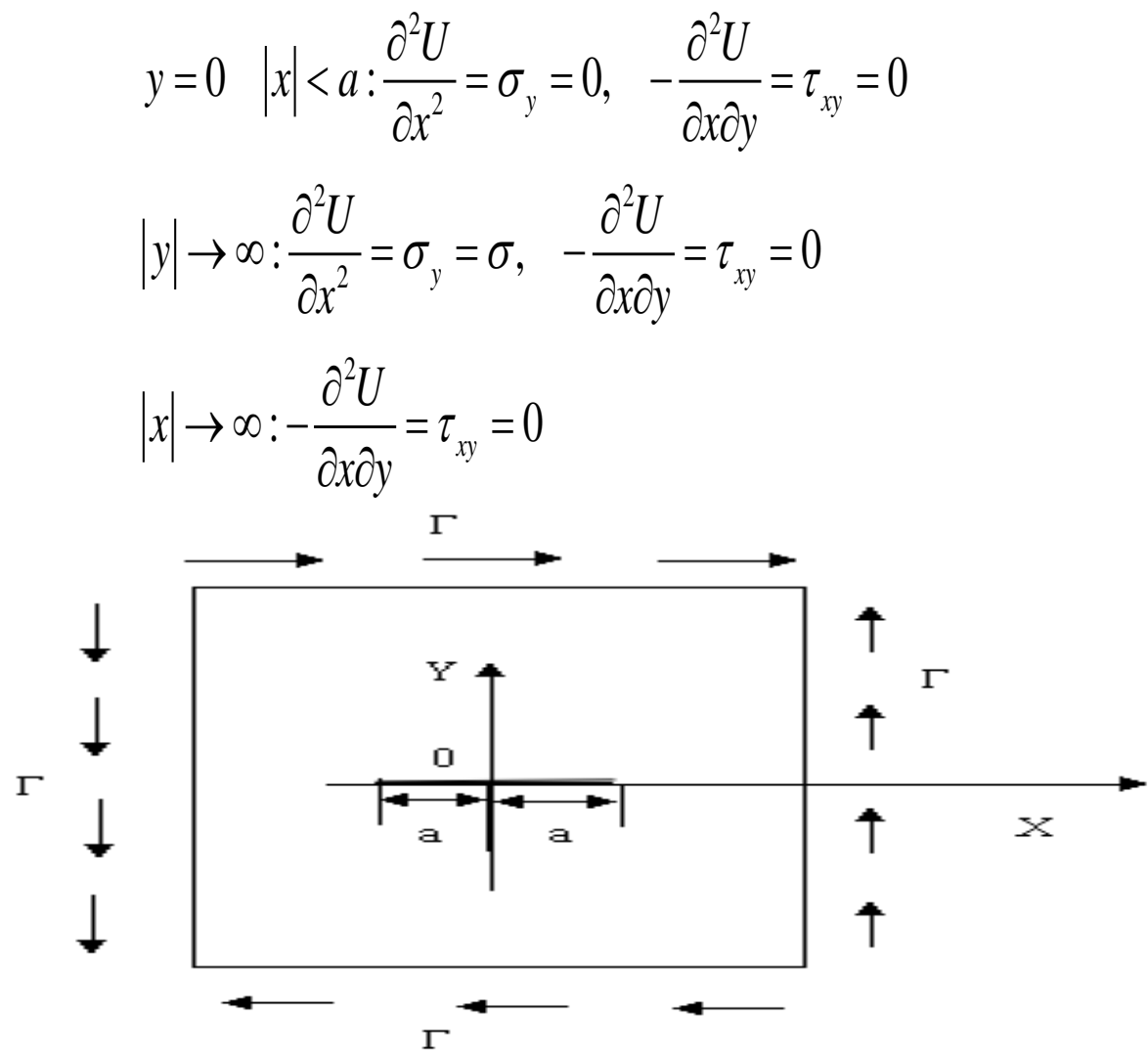

Fig.2 Plate with II mode crack

As shown in figure 2, the functionally graded material Plate with I mode crack which subjected to symmetric load $\tau$, its boundary conditions are as follows:

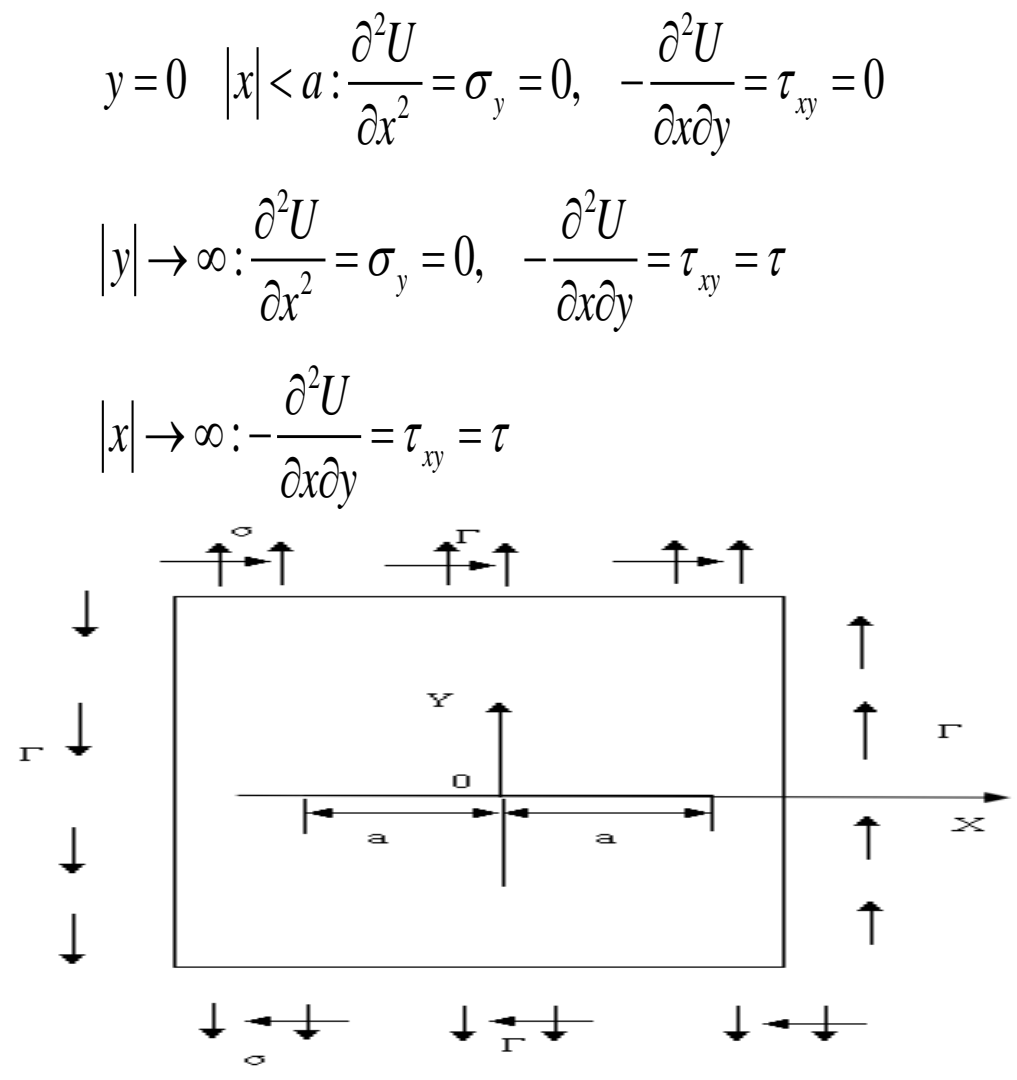

Fig.3 Plate with I+II mode crack 
As shown in figure3, the functionally graded material Plate with I mode crack which subjected to symmetric load $\sigma$ and $\tau$, its boundary conditions are as follows:

$$
\begin{aligned}
& y=0 \quad|x|<a: \frac{\partial^{2} U}{\partial x^{2}}=\sigma_{y}=0, \quad-\frac{\partial^{2} U}{\partial x \partial y}=\tau_{x y}=0 \\
& |y| \rightarrow \infty: \frac{\partial^{2} U}{\partial x^{2}}=\sigma_{y}=0, \quad-\frac{\partial^{2} U}{\partial x \partial y}=\tau_{x y}=\tau \\
& |x| \rightarrow \infty:-\frac{\partial^{2} U}{\partial x \partial y}=\tau_{x y}=\tau
\end{aligned}
$$

According to the basic equation and boundary condition, the discussion of isotropic functionally graded material plate I type, II type, type I + II attached to the crack tip recent fracture problem which will be summed up in solving three fourth order variable coefficient homogeneous linear partial differential equation boundary value problem.

\section{Acknowledgements}

This work was financially supported by The Project of the key discipline of theoretical physics of Xinxiang University.

\section{References}

[1] DELALE. F, ERDOGAN. F, The crack problem for a nonhomogeneous plane, ASME Journal of Applied Mechanics, vol. 50(1983)609-614.

[2] Sih. G. C, Liebowitz. H, Mathematical Theories of Brittle Fracture, Fracture, vol. 2(1968)108114.

[3] Corten. H. T, Fracture Mechanics of Composite, Fracture, vol. 7(1972)695- 703.

[4] Sih. G. C, Chen. E. P, Cracks in Composite Materials, Mechanics of Fracture, vol. 6(1981)1- 9.

[5] Yan. Li, Perspective and application of material phisics and chemistry in civil aviation, Ceramic science and art, vol. 12(2003)22-26.

[6] KONDA. N, ERDOGAN. F, The mixed mode crack problem in a nonhomogeneous elastic plane, Engineering Fracture Mechanics, vol. 47(1994)533-545. 